\title{
Do Early Birds Share Their Worms? How Prosocial Behavior and Empathy Vary Across the Day
}

\author{
Zoë Francis', Gregory Depow², Michael Inzlicht ${ }^{2}$ \\ ' University of the Fraser Valley \\ ${ }^{2}$ University of Toronto
}

November II, 2020

\section{Abstract}

Social behaviours have been proposed to follow circadian patterns across the course of the day, but empirical support is mixed. As a within-subject test of circadian effects on everyday behaviour, we conduct an experience-sampling study with a near-representative population of 285 Americans (7,405 observations). Prosocial behaviour and empathy were both somewhat more frequent in the morning ( $d$ $=0.08$ ). However, these non-linear patterns only described morning-type participants; evening-types had consistent (and lower) rates of prosocial behaviour and empathy across time-of-day. These patterns were explained by morning-types reporting more social opportunities earlier in the day. These findings suggest that small, within-subject diurnal effects on social behaviour occur in daily life, and that some circadian effects may be driven by situation selection.

Key words: prosocial behaviour, empathy, chronotype, individual differences, diurnal patterns, situation selection

\section{Notes}

This manuscript has been accepted for publication at the Journal of Research in Personality. This manuscript is a preprint, which may differ from the final published version.

Author Contributions. ZF suggested and preregistered the current analyses. GD, with assistance from ZF and $\mathrm{MI}$, designed the study, collected the data, and organized the data files. ZF analyzed the data and drafted the manuscript. GD and MI provided feedback and edits to the manuscript. All authors approved the final version of the submitted manuscript.

Funding. This work was supported by the Social Sciences and Humanities Research Council of Canada (435-20I9-0I44) to M. Inzlicht. 
Both feeling empathy and behaving prosocially help us build friendships, maintain relationships, and increase well-being. Some forms of prosocial behaviours, like the provision of social support, are even associated with reduced mortality (Brown et al., 2003). But people do not always choose to behave prosocially, nor always choose to empathize with others (Cameron et al., 2019; Lockwood et al., 2017). The likelihood of behaving prosocially varies by situational factors (Lefevor et al., 2017), individual differences (Habashi et al., 2016), and by internal states such as mood (Carlson et al., 1988). Recently, some have suggested that social decisions may also change across the course of the day (Kouchaki \& Smith, 20I4), alongside the subtle fluctuations in mood and cognition known as circadian rhythms or diurnal effects. While circadian rhythms are believed to affect people's self-selection into situations, their mood, and attention, the degree to which these fluctuations affect prosocial behaviours in daily life is not yet known. Here, we examine whether prosocial behaviours fluctuate across the day in everyday life - and whether these fluctuations are moderated depending on one's chronotype - by conducting an experience-sampling study with a near-representative' sample of American adults.

\section{Circadian and Synchrony Effects in the Lab}

Across the course of the day, attentional capacities, executive functioning, and mood each ebb and flow alongside physiological changes in hormone levels and body temperature (Schmidt et al., 2007). On average, attentional abilities are highest in the morning and decline as the day progresses (Riley et al., 2017). However, circadian rhythms are not consistent across people; they vary by one's chronotype. People with a morning chronotype prefer to wake and fall asleep earlier and are at their most alert earlier in the day. Those with evening chronotypes prefer to wake and fall asleep later, and reach peak alertness later in the day (Schmidt et al., 2007). Many circadian effects depend on chronotype. When tested at their preferred, optimal time-of-day (morning-types in the morning; evening-types in the evening), people are more alert on cognitive and academic tasks (Goldstein et al., 2007; Matchock \& Mordkoff, 2009), better maintain attention (Lara et al., 20 I4), are more aware of attempts to persuade them (Hossain \& Saini, 20l4), and make less stereotypic judgements (Bodenhausen, 1990). These matching or synchrony effects also apply to patterns of mood across the day. People with morning chronotypes experience their most positive moods in the morning, while those with evening chronotypes are happiest later in the day (Caminada \& Bruijn, 1992; Chebat \& Dubé, 1997; Kerkhof, 1998).

Given the fluctuating patterns of alertness and mood across the day, social psychologists have theorized that social decisions may also be affected by either overall circadian effects or by synchrony effects. Specifically, people may act more prosocially and morally during the morning (Kouchaki \& Smith, 20l4) or during the times that match their chronotype (synchrony effects; Gunia et al., 20I4). A growing number of studies have tested these hypotheses, with mixed results. Two experiments found that participants were less likely to lie or cheat in the morning, relative to the evenings - a finding termed the morning morality effect (Kouchaki \& Smith, 2014). Two similar studies found no main effects of time-of-day on cheating but instead found synchrony or matching effects, where morning-type

\footnotetext{
I The sample, described further in the methods section, was selected to be representative of the American population in regard to sex, age, ethnicity, education, region, and income. We use 'nearrepresentative' as shorthand, acknowledging that the sample is not necessarily reflective of the American population on other dimensions.
} 
participants were less likely to cheat in the morning and evening-type participants were less likely to cheat in the evening (Gunia et al., 20l4). Attempted replications, using various lab-based economic trust games, found no evidence of either circadian effects or synchrony effects on prosocial behaviour (Correa et al., 2017; Dickinson \& McElroy, 2017).

Other research has examined how prosocial behaviours might vary across time of day by taking advantage of natural variability in the time that people complete online tasks (Arechar et al., 2017) or allowing participants to complete the study at their choice of time-of-day (Study I and 2 in Kouchaki \& Smith, 2014; Roeser et al., 2016), again with inconsistent evidence for either main effects of circadian effects or for chronotype-synchrony effects. Thus, in controlled settings, it is still unclear whether or not prosocial behaviours are affected by either time-of-day or chronotype-synchrony.

\section{From the Lab to the Field}

Social behaviours in everyday life rarely resemble the economic games or lab-based tasks used to measure morality and prosociality in the lab. Rates of dishonesty in lab-based tasks do not resemble rates of dishonesty in comparable field studies (Gerlach et al., 2019), and the matrix task, often used by the above studies as a measure of dishonesty, may also be capturing honest mistakes in addition to intentional lies (Heyman et al., 2020). Recent studies have thus sought more ecologically valid evidence for circadian effects on prosocial behaviour, with surprising results. In contrast to the morning morality and synchrony effects inconsistently found in the lab, grocery store cashiers were more likely to overcharge customers in the morning ("morning immorality"; Vranka et al., 2019) and people waiting for a train were more likely to volunteer their time during times that were mis-matched with their chronotype (during their "off-times"; Solomon \& Zeitzer, 2019). Intriguingly, this initial evidence suggests that - in real life - people may be more likely to behave prosocially later in the day, rather than earlier in the day, and during times that are mismatched, rather than matched, to their chronotype.

A limitation to these between-subject studies - both in the field and those using correlational designs in the lab - is that they are necessarily conflating two possible mechanisms for time-of-day and synchrony effects: ( $I$ ) how circadian changes to cognition and affect influence social decision-making processes, and (2) how people who participate in a study during one time-of-day differ from those who participate during another time-of-day (Schofield, 2016). This second mechanism describes a selfselection effect.

\section{Self-Selection Effects}

Self-selection - where people's time-of-day preferences affect their likelihood of participating in a study at a given time - is typically considered a confounding variable, especially for correlational designs. Differences in who self-selects to sign-up for a study at different times of day impairs our ability to draw causal inferences about how time-of-day may affect decision-making. But selection effects can be even more impactful than commonly recognized. Even in experimental designs that attempt to randomize participant's time-of-day of participation, people are more likely to agree to participate and show-up during a preferred time than a non-preferred time (e.g., Gunia et al., 20I4), which can still lead to selective attrition effects and self-selection biases (Zhou \& Fishbach, 2016). 
Selection effects are not only relevant to study design - selection into and out of different situations or activities constantly occurs in everyday life, and is highly relevant for understanding human psychology and behaviour (Duckworth et al., 2016; Emmons \& Diener, 1986). In everyday life, people often have control over the situations they find themselves in, and can often influence whether or not they experience an opportunity to behave prosocially. Just like self-selection into psychology studies can be affected by circadian effects, people's actual patterns of social interactions (their opportunities to behave prosocially) also follow diurnal cycles (Hasler et al., 2008) and these opportunities may be further affected by chronotype. A morning-type person might be more likely than an evening-type person to agree to meet with friends for breakfast. An evening-type person may be more likely than a morning-type person to sign-up for an evening shift as a cashier in a grocery store. These situations may then provide opportunities for empathy and prosocial behaviour, whereas staying alone at home would not. While it is important to differentiate whether time-of-day effects on prosocial behaviour are due to selection effects or the direct effects of cognitive or affective fluctuations, both mechanisms might ultimately influence prosocial behaviour in everyday life.

\section{Motivation for Current Study}

Existing literature on the effects of circadian rhythms and chronotype on prosocial behaviours has been limited in multiple ways. While experimental methods are highly controlled, they may not reflect what actually occurs in everyday life, due to the limited generalizability of their measurements of prosociality and due to constrained operationalization of "morning" and "evening" time windows (Smith \& Kouchaki, 20I4). Correlational methods - including the field studies described above - cannot easily distinguish between selection effects and affective or cognitive effects on decision-making processes.

Furthermore, the reliance on any between-subject designs makes it difficult to conclude whether morality and prosociality vary across the day as a within-subject process; between-person and withinperson variability can be driven by distinct processes and have distinct relationships with relevant outcomes (Fisher et al., 2018). Ultimately, we are interested in whether people are more or less likely to act prosocially or engage in empathy at different times of day as a within-subject pattern of behaviour, and whether these within-subject fluctuations in prosociality depend on chronotype.

Finally, most prior research has been limited by under-powered, between-subject study designs. Given the inconsistent prior finding and thus potentially small effect sizes, high-powered, within-subject designs are required to determine whether circadian rhythms affect prosocial behavior.

In response, we report on an experience-sampling study that measured prosocial behavior and empathy experiences in everyday life. Experience-sampling - previously used to describe diurnal patterns in mood and self-control (Caminada \& Bruijn, 1992; Zhang et al., 2018) — allows us to have much higher statistical power, due to the within-subject design, more observations (over 7,000), and less constrained operationalizations of time-of-day. Furthermore, this methodology is not subject to between-subject selection effects that occur when participants choose a single time to complete the study (Schofield, 2016). We can instead measure real-life situation selection effects, by asking people to report on their opportunities to experience empathy or behave prosocially, alongside reporting on their actual empathy and prosocial behaviour. Finally, using experience-sampling methodology increases the generalizability and ecological validity of our findings, as participants are reporting on their behaviors and feelings in their real lives, in almost real-time. 


\section{Methods}

A (near) representative sample of Americans completed seven experience-sampling surveys per day for one week, and reported recent episodes of feeling empathy and prosocial behaviors (sample further described in Depow, Francis, \& Inzlicht, under review). Following preregistered R analysis scripts, available at rb.gy/7nimgw, we examined how these self-identified acts of both prosociality and empathy vary across time-of-day, and how these patterns differ according to the participant's chronotype ${ }^{2}$. We examined three possible explanatory variables - subjective well-being and subjective difficulty of empathy (affective mechanisms), and opportunities to engage in empathy (situation self-selection).

Data files are available at rb.gy/7nimgw. This experience-sampling study on empathy in daily life was reviewed and approved by the institutional review board. The same dataset is used in Depow, Francis, \& Inzlicht (under review).

\section{Participants}

The sample for this study was pre-screened to be representative of the American population, using quota sampling conducted by the survey company Qualtrics. First, Qualtrics had 3,486 potential participants from their panel complete a demographics questionnaire and attention check. Of this pool, $84 \mathrm{I}$ individuals were selected to match American census data on the characteristics of sex, age, ethnicity, education, region, and income, and were emailed a baseline survey. This baseline survey was completed by 376 people, and 285 of those went on to download the experience-sampling app and complete at least one experience-sampling survey.

Our final sample $(N=285)$ was close to representative, albeit with both Latino participants and those making over $\$ 100,000$ underrepresented. We had participants from all age groups (5\% from $18-24$, $24 \%$ from $25-34,24 \%$ from $35-44,21 \%$ from $45-54$, I $7 \%$ from $55-64$, and $9 \%$ over 65 ) and $54 \%$ identified as female. 194 participants identified as White/Caucasian (non-Hispanic), 36 as African American, 25 participants as Hispanic, 13 as Asian American, and 5 as other. Participants had varying household income, education levels, and were from various geographical areas of the US.

Participants completed an average of 26.I (SD = 14.6) surveys across the week, with a median of 30 surveys. We included all participants who completed the baseline survey (which included the chronotype measure) and at least one experience-sampling survey in our analyses, following our preregistration. One participant did not complete the chronotype measure. Overall, we had 7,405 experience-sampling surveys available for analysis.

We did not have full control over the sample size, given unpredictable attrition between the intake survey and the experience-sampling surveys, but had preregistered a minimum of $N=250$. An estimated power analysis, conducted with G*Power (Faul et al., 2009), suggests that $N=285$ and 25 repeated measures per participant should have $80 \%$ power to detect a between-within interaction

\footnotetext{
${ }^{2}$ Following our preregistration, we also tested whether time-of-day patterns would differ according to implicit theory of willpower (Job et al., 2010). Preregistered analyses involving willpower theory were not generally significant and are available in the Supplemental Materials.
} 
effect of $d=.10$ (repeated-measures $r=.50$ ). This sample size should also have sufficient power to model nonlinear cosinor effects (Zhang et al., 20l8).

\section{Procedure}

Baseline Measures. After completing a screening procedure (conducted by Qualtrics panels, consisting of an attention check question and demographic questions), being invited to participate, and providing informed consent, participants completed a set of baseline questionnaires including the fiveitem reduced Morningness-Eveningness Questionnaire (rMEQ) to measure chronotype ( $\alpha=.7 \mathrm{I}, 95 \%$ $\mathrm{Cl}[.66, .75]$; Adan \& Almirall, 199I), as well as other questionnaires not examined here (see Supplementary materials for discussion of willpower theory scale).

Experience-sampling Surveys. Starting the following day, participants received seven short surveys per day, sent between I0am and I0pm EST, delivered by Metricwire. Surveys were sent on a random schedule with an average of 105 minutes between surveys, and a minimum of 30 minutes between one survey and the next. Because participants were from all five American time-zones (GMT-10, $-7,-6$, -5 , and -4 ), we thus had some experience-sampling surveys completed between 5am to I $2 \mathrm{am}$. Most surveys (95\%) were completed between 9am and 10pm local time, and we thus only draw conclusions and graphs within this time window. We used local time (adjusted for participants' time zones) for all analyses.

After receiving a survey prompt, participants had one hour to respond to the survey on the smartphone app. Participants first responded to two questions about their subjective well-being $(\mathrm{I}-7$, "At the moment, how happy do you feel?" and, "At the moment, do you feel that your life has a clear sense of purpose?"). They then indicated whether or not they had performed a prosocial behavior, "In the last 15 minutes, did you do anything to directly or indirectly help, or make another person feel better? (e.g. by offering comfort, financial support, advice or assistance)" (Myes $=20.1 \%$ of surveys).

Participants next answered whether, in the prior 15 minutes, they had had an opportunity to feel empathy for someone ( $M_{\text {yes }}=19.2 \%$ of surveys). If they responded "no", participants later saw a question asking them if they had "seen someone express an emotion" in the prior 15 minutes $\left(M_{\text {yes }}=\right.$ $9.4 \%$ when question was asked; $7.6 \%$ of all surveys). Participants who responded "yes" to either question were coded as having had an opportunity to empathize. Note that our preregistration stated that we would only use the first question as indicating an 'opportunity to empathize'. However, participants who saw someone express an emotion also did have an opportunity to empathize or behave prosocially, even if they did not perceive it as such.

Participants then indicated whether or not they had actually felt empathy for someone $\left(M_{\text {yes }}=16.9 \%\right.$ of all surveys; $87.9 \%$ when opportunity available). They were then asked, when applicable, about the subjective difficulty of engaging in each of three components of empathy - emotion sharing, perspective-taking, and generating compassion - on I to 7 scales, which were then averaged. The survey length was consistent regardless of participants' answers. Other questions (including "filler" questions), not analyzed here, are described in Depow, Francis, \& Inzlicht (under review).

Analysis Plan. Following our preregistered analysis plan, we conducted three-level hierarchical models, with experience-sampling surveys nested within days, nested within participant (Equation I). 
Our preregistered analyses included testing the fixed linear effects of time-of-day $\left(T_{i j k}\right)$, chronotype $\left(\mathrm{Chro}_{i}\right)$, and the chronotype-by-time interaction term $\left(T_{i j k} C h r o_{i}\right)$. Chronotype was grand-mean centered and tested as a continuous variable. We included random intercepts for participant $\left(P_{\mathrm{i}}\right)$ and day $\left(P D_{\mathrm{ij}}\right)$. We preregistered four dependent variables: the binomial variables (yes $=1$, no $=0$ ) of prosociality, empathy, and the opportunity to experience empathy, and the continuous variable of perceived empathy difficulty. For the three binomial dependent variables, we conducted generalized linear models, specifying a binomial distribution with a log link. Because empathy difficulty was highly rightskewed $($ median $=1.33$, I to 7 scales), we also analyzed this dependent variable with a generalized linear model, instead specifying a gamma distribution. To interpret significant interactions, we examined the simple slopes as per Preacher, Curran, \& Bauer (2004).

Eq. I: $Y_{i j k}=\beta_{0}+\beta_{1} T_{i j k}+\beta_{2}$ Chro $_{i}+\beta_{3}$ Chro $_{i} T_{i j k}+P_{i}+P D_{i j}+\varepsilon_{i j k}$

To facilitate comparison between the effect sizes of our findings and effect sizes reported by prior literature (which typically have approximately 9 hours between the "morning" and "evening" time slots), we report Cohen's d reflecting the change expected from a nine-hour difference. Our analyses otherwise treated time as a continuous variable.

Because time-of-day effects are frequently not linear, we also report additional analyses using a cosinor fitting procedure (following Zhang et al., 2018). To test nonlinear diurnal patterns, time-of-day is converted into radians (divided by 12 , multiplied by $2 \pi$ ) and transformed into two component variables - sine and cosine of time - that together model the strength of the diurnal pattern. Using two sinusoidal components allows for nonsymmetric curves and better captures behaviors that may peak at different times-of-day; a sine wave across the 24-hour day best captures behaviors that peak at 6am, while a cosine wave best captures behaviors that peaks at I 2 am (Hasler et al., 2008). We tested the significance of the overall diurnal pattern by comparing a multilevel model including these two transformed time-of-day variables (Equation 3) against a null model including only the random intercepts for participant and participant day, and no fixed predictors (Equation 2).

Eq. 2: $Y_{i j k}=\beta_{0}+P_{i}+P D_{i j}+\varepsilon_{i j k}$

Eq. 3: $Y_{i j k}=\beta_{0}+\beta_{1} \sin T_{i j k}+\beta_{2} \cos T_{i j k}+P_{i}+P D_{i j}+\varepsilon_{i j k}$

To see whether the diurnal pattern differed by chronotype, we then compared a model with only the main effects of chronotype and time (sine and cosine; Equation 4) against a model that included interaction terms between chronotype and both diurnal variables (Equation 5).

Eq. 4: $Y_{i j k}=\beta_{0}+\beta_{1} \operatorname{sinT} T_{i j k}+\beta_{2} \cos T_{i j k}+\beta_{3} \operatorname{Chro}_{i}+P_{i}+P D_{i j}+\varepsilon_{i j k}$

Eq.5: $Y_{i j k}=\beta_{0}+\beta_{1} \operatorname{sinT} T_{i j k}+\beta_{2} \cos T_{i j k}+\beta_{3} \operatorname{Chro}_{i}+\beta_{4} \sin T_{i j k} \operatorname{Chro}_{i}+\beta_{5} \cos _{i j k} \operatorname{Chro}_{i}+P_{i}+P D_{i j}+\varepsilon_{i j k}$

Diurnal patterns are described by both their amplitude and the phase shift (Zhang et al., 20I8). The amplitude is maximum deviation of the sinusoidal wave from the mean, reflecting the strength or magnitude of the diurnal pattern. The phase shift reflects the time-of-day of the sinusoidal maximum.

These characteristics are independent. 
Eq. 6: $A m p=\sqrt{\beta_{1}^{2}+\beta_{2}^{2}}$

Eq. 7: Phase shift $=\left\{\begin{array}{lr}\tan ^{-1}\left(\frac{\beta_{1}}{\beta_{2}}\right)+\pi & I F \beta_{2}<0 \\ \tan ^{-1}\left(\frac{\beta_{1}}{\beta_{2}}\right) & I F \beta_{2}>0 \text { AND } \beta_{1}>0 \\ \tan ^{-1}\left(\frac{\beta_{1}}{\beta_{2}}\right)+2 \pi & \text { IF } \beta_{2}>0 \text { AND } \beta_{1}<0\end{array}\right.$

\section{Results}

\section{Prosociality and Empathy Across the Day (Preregistered Linear Effects)}

We first report our preregistered analyses examining the main linear effects of time of day, chronotype, and their interaction.

Prosocial Behavior. Overall, the likelihood of prosocial behaviour did not change linearly across the day $(d=-0.08, z(7399)=-1.68, p=.092)$. However, the likelihood of prosocial behaviour did change across time-of-day depending on chronotype, albeit with a relatively small effect size (Figure IA; interaction $d=-0.12, z(7399)=-2.05, p=.040)$. Those with a more morning chronotype $(-I S D)$ were more likely to report prosocial behavior earlier in the day, and less likely to report prosocial behavior as the day progressed $(d=-0.18, z(7399)=-2.76, p=.006)$. Those with a more evening chronotype (+ISD) were no more or less likely to report prosocial behavior depending on time-of-day $(d=0.02, z(7399)=0.25, p=.799)$. On average (i.e., at the day's midpoint), those with more morning chronotypes were somewhat more likely to report prosocial behaviour, although the effect size was again quite small $(d=.12, b=0.21, S E=0.10, z(7399)=1.98, p=.048)$.

Experiencing Empathy. The same pattern seen for prosocial behaviors was also true for reports of feeling empathy for another - unsurprising, given that reports of empathy frequently cooccurred with reports of prosocial behavior $(r=.70, t(744 I)=85.02, p<.001)$. While the linear effect of time-of-day again was not significant as a main effect $(d=-0.08, z(7399)=-1.67, p=.095)$, there was a significant, albeit small, moderation by chronotype (Figure IB; $d=-0.12, z(7399)=-2.22, p=.026, r=-0.0 \mathrm{I}$ ). Morning-type participants were more likely to report feeling empathy in the morning and less likely to report feeling empathy in the evening (at $-I S D, d=-0.20, z(7399)=-2.84, p=.004$ ), while eveningtype participants were not any more or less likely to feel empathy at any particular time of day (at + ISD, $d=0.02, z(7399)=0.38, p=.705)$. Unlike for prosocial behavior, there was no main effect of chronotype $(d=0.06, z(7399)=1.09, p=.276)$, in that morning-type participants were no more likely to empathize at the day's midpoint. 
Figure I. Linear effects of time-of-day on prosocial behaviour and empathy by chronotype
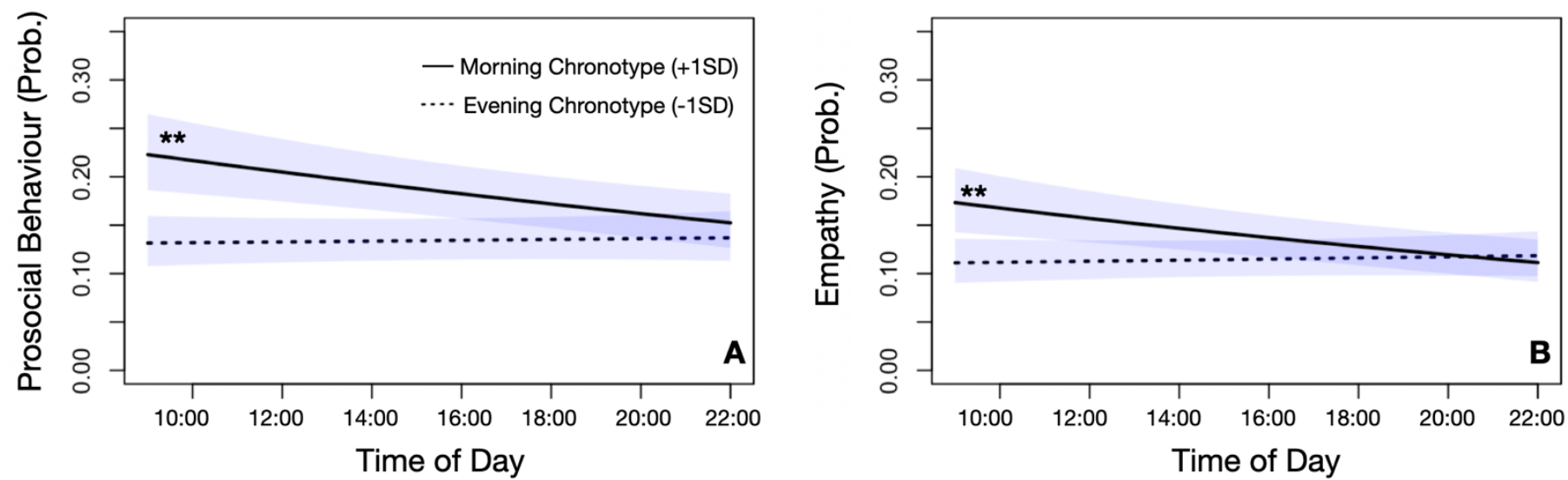

Note. Figures show effects based on multi-level models, modelling the linear effects of time-of-day and their interactions with chronotype (shown at +ISD and -ISD). Ribbons show standard error for the effect of chronotype. Non-overlapping ribbons signify statistically significant simple effects of chronotype at that time of day. Simple effects of time-of-day are shown for morning- and eveningtypes, $*=p<.05 ; * *=p<.01$.

\section{Cosinor Diurnal Patterns of Prosociality and Empathy}

Because time-of-day patterns are not usually best explained by linear effects, we also examined the effects of time using cosinor diurnal modelling. We further tested whether cosinor diurnal patterns were moderated by chronotype.

Prosocial Behavior. The first analysis, comparing the null model to a model with the diurnal pattern (the cosine and sine of time-of-day), found that the likelihood of prosocial behavior followed a cosinor pattern $\left(\chi^{2}(3,5)=8.03, p=.018\right)$. This diurnal model also outperformed the preregistered linear timeof-day model of prosocial behavior, presented above $\left(\chi^{2}(4,5)=4.59, p=.03\right)$, suggesting that this nonlinear pattern better describes the variability in prosocial behavior across the course of the day.

To test whether the diurnal pattern varied by chronotype, we compared a model with the diurnal pattern components and chronotype to a model with the diurnal pattern components, chronotype, and their interaction. The moderation of the diurnal pattern by chronotype was not significant (Figure 2A; $\left.\chi^{2}(6,8)=4.07, p=.131\right)$, suggesting that the cosinor time-of-day pattern of prosocial behavior did not differ significantly for those with morning- versus evening-chronotypes.

Empathy. Empathy likelihood also could be explained by a cosinor pattern $\left(\chi^{2}(3,5)=6.00, p=.0499\right)$, though the diurnal model did not fit the empathy data significantly better than the linear model $\left(\chi^{2}(4,5)\right.$ $=2.66, p=.102)$. Like the linear time-of-day model, the diurnal pattern of empathy varied significantly by chronotype (Figure $2 \mathrm{~B} ; \chi^{2}(6,8)=7.75, p=.021$ ). Changes in the likelihood of empathy were much larger for participants with morning chronotypes $(-I S D, A m p=.4 I)$, relative to evening-types $(+I S D$, $A m p=.05)$. 
Figure 2. Cosinor diurnal patterns of prosocial behaviour and empathy by chronotype
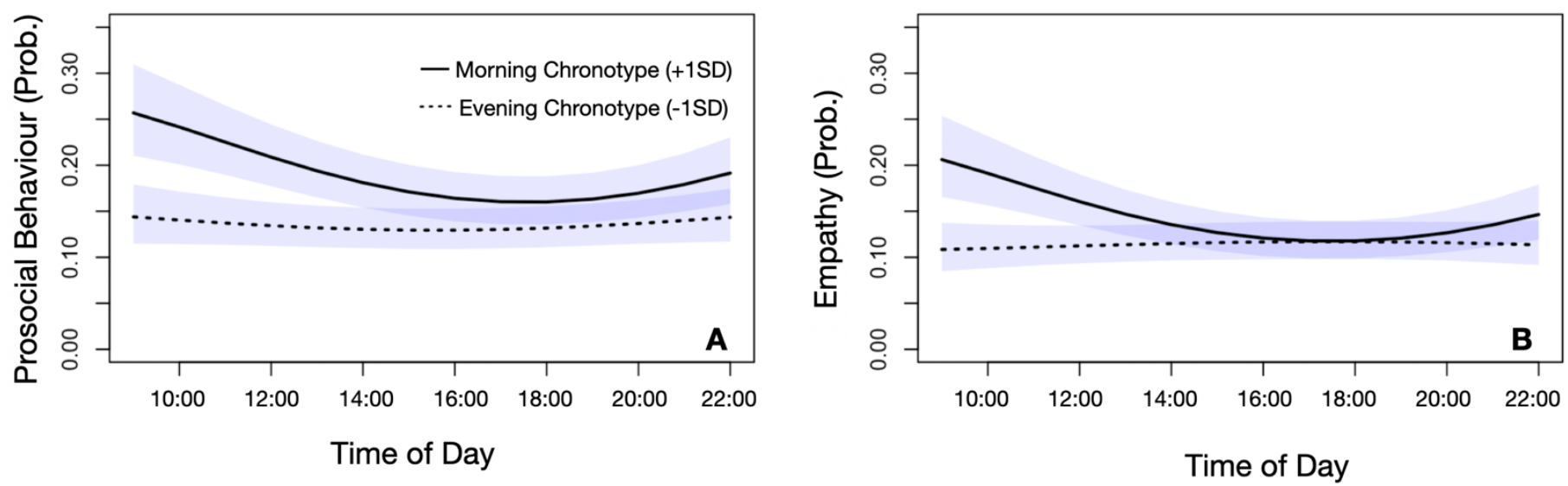

Note. Ribbons show standard error for the effect of chronotype. Non-overlapping ribbons signify a significant simple effect of chronotype at that time of day.

\section{Possible Explanatory Variables}

Why are more morning-types more likely to be empathetic and prosocial in the mornings? We examined whether, in the morning hours, morning-types were (i) more likely to have been in contact with other people (having the opportunity to empathize) (ii) were more likely to find empathy easier, or (iii) were in more positive moods. Mood was not preregistered as a variable of interest, but existing daily diary research suggests that people are more likely to empathize when they are in a more positive mood, as a within-subject effect (Nezlek et al., 200I).

Opportunity to Empathize. Morning participants might be engaging in empathy more frequently in the mornings solely because they are more likely to have the opportunity to do so. We thus examined whether participants in general - or just more morning-types - were more likely to see another person's emotion in the morning.

First, we conducted our preregistered linear analyses. There was no significant main effect of either chronotype $(d=0.02, z(7399)=0.40, p=.686)$ nor of linear time-of-day $(d=-0.04, z(7399)=-0.96$, $p=.338$ ), and the interaction between chronotype and linear time-of-day was not significant at $\alpha=.05$ (Figure 3A; $d=-0.10, z(7399)=-1.90, p=.058)$. Both the linear pattern and effect size of this interaction, however, is comparable to that seen for prosocial behavior and empathy, with morningtype participants being somewhat more likely than evening-type participants to report opportunities to empathize in the morning. 
Figure 3. Linear effects of time-of-day on social opportunities and happiness by chronotype
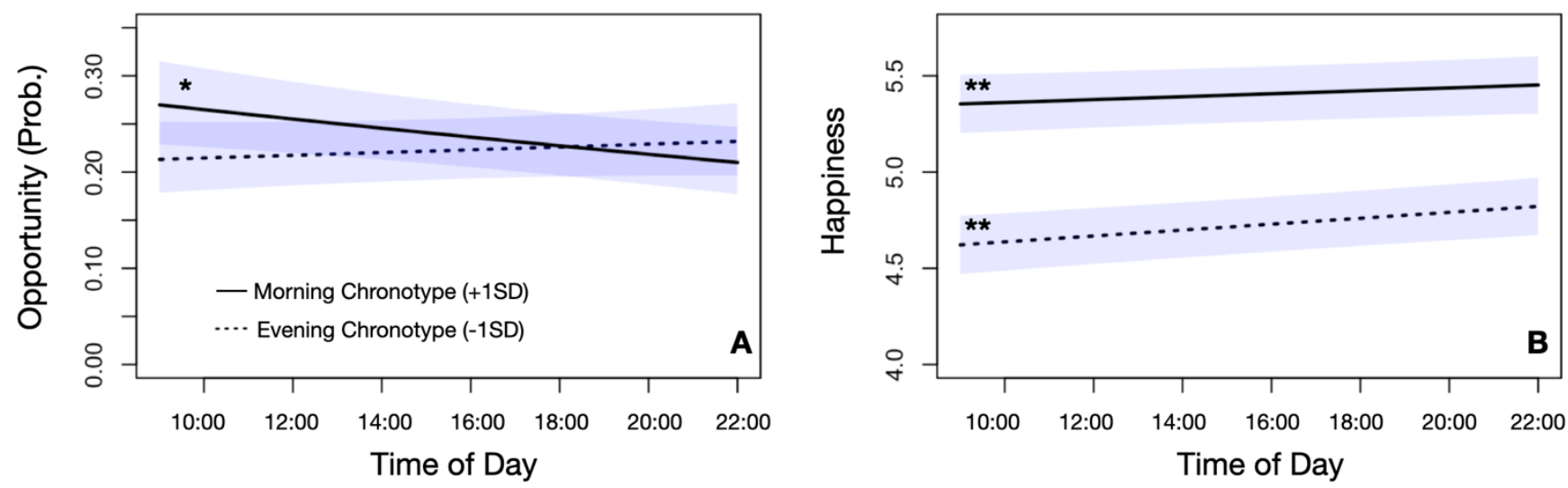

Note. Ribbons show standard error for the effect of chronotype. Non-overlapping ribbons signify a significant simple effect of chronotype at that time of day. Simple effects of time-of-day are shown for morning- and evening-types, $*=p<.05 ; * *=p<.01$.

Next, we conducted the parallel nonlinear analyses, using cosinor modelling. Before considering chronotype, opportunities to empathize were not significantly explained by a diurnal pattern $\left(\chi^{2}(3,5)=\right.$ 1.69, $p=.429)$. However, there was a significant interaction between the cosinor time variables and trait chronotype (Figure $4 \mathrm{~A} ; \chi^{2}(6,8)=7.89, p=.019$ ), such that the diurnal pattern was weaker for evening-types (at -ISD, Amp =.I5) and significantly stronger for morning-types (+ISD, Amp =.27). In addition to a more morning chronotype being associated with larger fluctuations in opportunities across the day, peak times also varied by chronotype: morning chronotypes reported the most opportunities early in the morning, while evening chronotypes reported the most opportunities in late afternoon (Figure 4A).

Across analyses, opportunity to empathize did not significantly change across time-of-day as a main effect (neither as a linear nor cosinor effect) but reported empathy opportunities changed across timeof-day more for those with morning chronotypes.

Figure 4. Diurnal patterns of time-of-day on social opportunities and happiness by chronotype
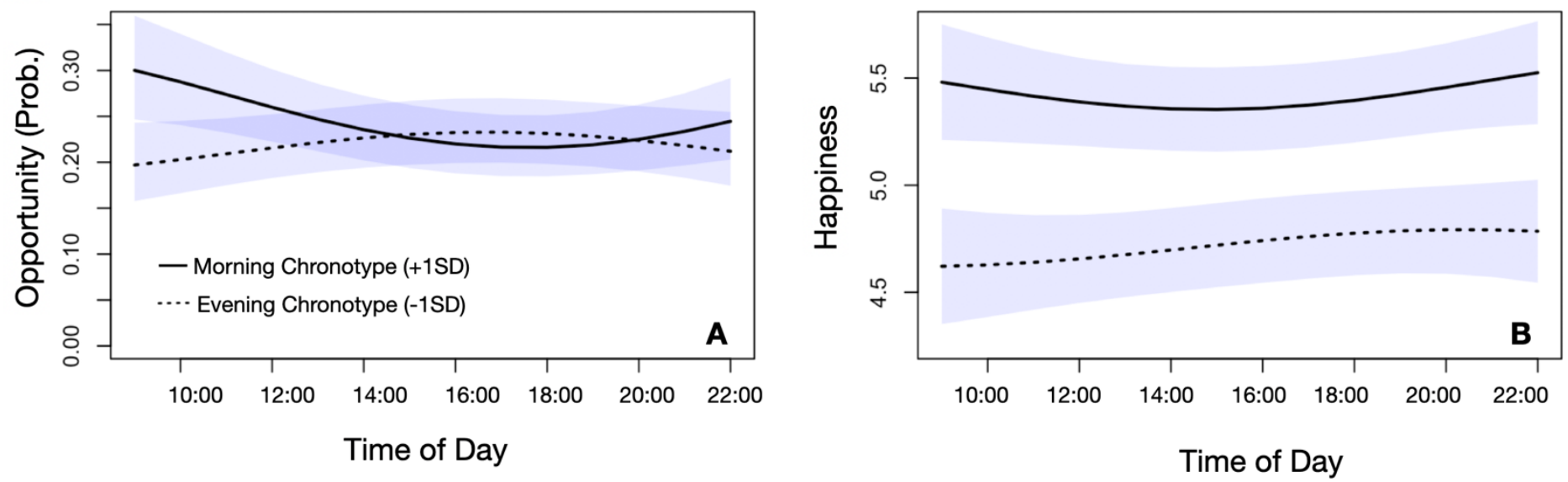

Note. Ribbons show standard error for the effect of chronotype. Non-overlapping ribbons signify a significant simple effect of chronotype at that time of day. 
Empathy Difficulty. Participants may be less likely to engage in empathy when it feels more difficult to do so. Engaging in empathy was reported as being slightly more difficult later in the day relative to earlier in the day $(d=0.02, z(1234)=2.5 \mathrm{I}, p=.012)$. This effect was not moderated by chronotype $(d$ $<0.01, z(I 234)=-0.36, p=.717)$. There was also no main effect of chronotype $(d<0.01, z(1234)=$ $0.30, p=.766)$.

We also conducted these analyses looking at the cosinor patterns of time-of-day. Again, the perceived difficulty of empathizing followed a diurnal pattern $\left(\chi^{2}(3,5)=9.00, p=.01 ; A m p=0.03\right.$, $\left.\max =7: 33 p m\right)$, but did not significantly depend on chronotype $\left(\chi^{2}(6,8)=1.63, p=.442\right)$. Across both linear and cosinor analyses, empathy was perceived as more difficult as the day progressed, regardless of chronotype. Although perceptions of empathy as difficult did not vary by chronotype, participants did tend to report that empathy felt more difficult later in the day compared to earlier in the day, as a within-subject effect.

Happiness. Finally, we examined subjective happiness as a third potential explanatory variable. As a linear test, participants generally reported more happiness as the day progressed $(d=0.08, t(6427)=$ $3.32, p<.001)$. Participants with more morning-type chronotypes were also happier overall than participants with more evening chronotypes $(d=.57, t(278)=4.75, p<.00 \mathrm{I})$; this main effect of chronotype was much larger than any diurnal effects or moderations observed in this study.

Chronotype was not a significant moderator of the linear time-of-day effect on mood (Figure 3B; $d=$ $-0.03, t(6500)=-1.09, p=.277)$.

Happiness likewise varied across time-of-day in a nonlinear fashion $\left(\chi^{2}(3,5)=13.37, p=0.00 \mathrm{I}\right)$. Mirroring the chronotype moderation found when modelling time as linear (above), the nonlinear diurnal pattern of happiness was also moderated by chronotype (Figure 4B; $\chi^{2}(6,8)=6.91, p=.032$ ). While both morning and evening chronotypes had a similar strength to their diurnal patterns $($ Amp $=$ .09 for evening-type, $A m p=.13$ for morning-type), their peak moods were at different times, with the models predicting peak happiness for morning-type participants (+ISD) in the early morning, and peak happiness for evening-type participants (-ISD) around 8:30pm.

\section{Effects on Prosocial Behavior, Above and Beyond Opportunities to Empathize?}

Two potential explanatory variables - opportunities to empathize and happiness - varied across timeof-day differently depending on chronotypes, like empathy and prosocial behavior themselves. We thus investigated whether the effects of time-of-day on empathy and prosocial behavior held after controlling for these explanatory variables (Table I). Because perceptions of empathy difficulty were only measured on surveys when participants reported engaging in empathy, we could not conduct the equivalent analysis controlling for empathy difficulty.

Overall, most observed circadian patterns in prosocial behavior and empathy could be explained by whether or not participants had had an opportunity to empathize. After accounting for empathy opportunities, none of the moderations of time-of-day by chronotype remained significant (Table I). 
Most of the main effects of time-of-day were also accounted for by opportunities to empathize ${ }^{3}$. Controlling for mood, on the other hand, did not reduce the previously observed temporal effects. These analyses suggest that the observed patterns in prosocial behavior and empathy - particularly the patterns that varied by chronotype - are not due to circadian shifts in mood, but are likely due to whether or not participants had interacted with others.

Table I. Model summary statistics

\begin{tabular}{|c|c|c|c|}
\hline Model & $\begin{array}{l}\text { No control } \\
\text { variable }\end{array}$ & $\begin{array}{l}\text { Controlling for } \\
\text { Empathy } \\
\text { Opportunity }\end{array}$ & $\begin{array}{l}\text { Controlling for } \\
\text { Mood }\end{array}$ \\
\hline \multicolumn{4}{|l|}{$\begin{array}{l}\text { I. Predicting prosocial } \\
\text { behavior }\end{array}$} \\
\hline Linear time-of-day effect & $-0.016(0.010)+$ & $-0.011(0.012)$ & $-0.019(0.010)^{*}$ \\
\hline Trait Chronotype & $0.206(0.104)^{*}$ & $0.224(0.087)^{*}$ & $0.215(0.105)^{*}$ \\
\hline $\begin{array}{l}\text { Linear time-of-day by chronotype } \\
\text { interaction }\end{array}$ & $-0.023(0.0 \mathrm{II})^{*}$ & $-0.013(0.014)$ & $-0.023(0.011)^{*}$ \\
\hline Empathy Opportunity & - & $3.66(0.1 \mathrm{I})^{* * *}$ & - \\
\hline \multicolumn{4}{|c|}{ II. Predicting empathy engagement } \\
\hline Linear time-of-day effect & $-0.017(0.010)+$ & $-0.02 I(0.015)$ & $-0.020(0.010)^{*}$ \\
\hline Trait Chronotype & $0.114(0.104)$ & $0.170(0.099)+$ & $0.120(0.105)$ \\
\hline $\begin{array}{l}\text { Linear time-of-day by chronotype } \\
\text { interaction }\end{array}$ & $-0.026(0.012)^{*}$ & $-0.015(0.017)$ & $-0.026(0.012)^{*}$ \\
\hline Empathy Opportunity & - & $22.20(630.36)^{\mathrm{a}}$ & - \\
\hline Mood & - & - & $0.26(0.03)^{* * *}$ \\
\hline & $\chi^{2}(\mathrm{df}=2)$ & $\chi^{2}(\mathrm{df}=2)$ & $\chi^{2}(\mathrm{df}=2)$ \\
\hline \multicolumn{4}{|l|}{ III. Predicting prosocial behavior } \\
\hline Cosinor time-of-day effect & $8.03 *$ & $6.62 *$ & $9.17 *$ \\
\hline $\begin{array}{l}\text { Cosinor time-of-day by } \\
\text { chronotype interactions } \\
\text { IV. Predicting empathy engag }\end{array}$ & 4.07 & 0.51 & 3.59 \\
\hline Cosinor time-of-day effect & $6.00^{*}$ & 4.04 & $6.65 *$ \\
\hline $\begin{array}{l}\text { Cosinor time-of-day by } \\
\text { chronotype interactions }\end{array}$ & $7.75^{*}$ & 1.02 & $6.91 *$ \\
\hline
\end{tabular}

Note. ${ }^{a}$ Engaging in empathy was only possible when there was an empathy opportunity. The dependency between these variables means that this standard error may be less accurate. $+=.05<p$ $<.10 ; *=.01<p<.05$.

\footnotetext{
${ }^{3}$ Alternatively, we can conduct analyses on the subset of the data (1994 observations) where an empathy opportunity was available. This analysis strategy results in the same conclusion, with trait chronotype predicting prosocial behaviour as a main effect $(b=0.27, S E=0.10, z(1983)=2.73, p=.006)$ and marginally predicting empathy $(b=0.17, S E=0.10, z(1983)=$ I.69, $p=.090$ ), and with no significant effects of time-of-day nor interactions on either dependent variable (ps $>.18$ ).
} 


\section{Discussion}

In a near-representative American sample, patterns of both prosocial behavior and empathy in daily life revealed a small, nonlinear time-of-day effect. Overall, participants were I.I 6 times more likely to behave prosocially earlier in the day compared to nine hours later, corresponding to a Cohen's $d$ of 0.08. But these changes to empathy and prosociality across time-of-day were not equally shown by everyone. "Morning larks" - those who rise earlier and feel at their best earlier in the day - tended to be more prosocial and empathetic in the morning, while "evening owls" were no more likely to be prosocial or empathetic in either the morning or evening. Furthermore, patterns of prosocial and empathetic behaviors did not decline across the day in a linear fashion but instead fluctuated nonlinearly, with prosocial behaviour being most likely in the early morning, least likely around dinner time, and increasing again as the evening progressed.

These diurnal patterns of prosocial behavior and empathy were best explained not by fluctuations in mood or perceived difficulty of empathizing, but by people's available opportunities. Morning-types were more likely to report seeing another person experience an emotion in the mornings, while evening-type people were, if anything, slightly more likely to come into contact with others' emotions in the afternoon. These seem to be real-life "selection effects" - people may be putting themselves in social situations more often during times that they feel awake and attentive. The same process seems to explain why participants were less likely to engage in empathy or prosocial acts on Saturdays (see Supplemental Materials) - they were less likely to have the opportunity.

Interestingly, evening-chronotypes were not especially likely to behave prosocially or engage in empathy later in the day. The lack of cross-over effect is consistent with other research finding that morning chronotypes benefit from their optimal time more than evening chronotypes benefit from theirs (Nowack \& Van Der Meer, 2018). The lack of cross-over also meant that morning-types were more likely to engage in prosocial behavior overall, consistent with previously described associations between evening chronotypes and higher impulsivity, aggression (Hwang et al., 2016), anxiety, and depression (Park et al., 2015).

\section{Implications}

This research provides a new perspective on how chronotype and circadian effects might affect prosocial behaviour in the real world, by showing small but significant effects of both circadian effects and (for morning chronotypes) synchrony effects on prosocial behaviour and empathy. These findings may provide more context for understanding individual differences in prosocial behaviour, and be relevant to individual difference researchers who are concerned about the impact of time-of-day effects on peoples' momentary behaviours.

Second, the non-linearity of the observed effects is critical for both interpreting past work on circadian effects and for planning future research. Prior research on 'morning morality' and diurnal effects have often compared inconsistent windows of the day (e.g., comparing morning to afternoon, versus comparing morning to evening, versus comparing day to night; Smith \& Kouchaki, 20I4). Because timeof-day effects are non-linear, it is unsurprising that prior tests have had heterogenous results - we would expect a greater difference when comparing $10 \mathrm{am}$ to $6 \mathrm{pm}$, and a smaller difference when 
comparing 10am to 10pm (Figure 2). Researchers interested in circadian effects should ensure they precisely operationalize the time frames of their predictions and not assume linearity (Scholz, 20 I9).

More generally, this research demonstrates how seemingly mundane explanations for behaviour having or not having the opportunity to feel empathy or behave prosocially - can itself be affected by trait chronotype. Individual differences do not only affect people's decisions and behaviours once they are in a given situation, but also affect the likelihood of experiencing that situation (Emmons \& Diener, 1986; Rauthmann et al., 20I5). While these self-selection effects can sometimes impede our ability to study more "psychologically interesting" processes like the effects of mood or cognition on behaviour, the current findings suggest that self-selection effects can be more predictive of actual behaviours in daily life.

This study further demonstrates how one trait, chronotype, can affect situation self-selection and behaviours that are not core to our understanding of the trait itself. Often, a trait will affect situation self-selection and resulting behaviours that are definitionally related to the trait: conscientiousness affects exposure to dutiful situations, openness affects exposure to intellectual situations, and trait selfcontrol affects exposure to self-control dilemmas (Duckworth et al., 2016; Rauthmann et al., 20I5). Chronotype, on the other hand, a trait about time-of-day preferences seems to predict people's experience of social opportunities, perhaps even incidentally. It is possible that evening-type people did not intend to avoid social interactions in the morning, specifically; instead, the lack of social opportunities may be a side effect of evening-type people's slow start to their day or general lack of attention in the morning hours.

\section{Limitations and Future Directions}

While our experience-sampling data spanned from 9am to II pm, we did not collect data from the late evening or early morning. We are thus missing data from times where night owls might be prosocial or more likely to emphasize, when the morning larks are asleep. By not sampling across all hours, we were also missing data that may have made our diurnal models more accurate - it is probably not the case that morning-chronotypes are maximally likely to be prosocial at 5:30am. The current data best reflects people's experiences during the daytime and evening hours, the hours when most activity in society and social interactions occur.

Furthermore, participants did not complete all experience-sampling surveys. However, completed experience-sampling surveys do seem to be representative of participants' own daily lives (Sun, Rhemtulla, \& Vazire, 2019), suggesting that our results should be approximately generalizable to Americans who own smartphones and are willing to complete experience-sampling surveys. Our results may not generalize to other countries or cultures.

Like other experience-sampling studies, this study relied on self-report measures (Scollon et al., 2003). We cannot be sure that different people were using the same definitions of prosocial behaviors or feelings of empathy, despite our attempts to define these concepts to participants at the beginning of the study. Variability in definitions and reporting could influence our tests of between-subject factors (chronotype). Furthermore, we were not able to distinguish between different types of prosocial activities; it is possible that some types of prosocial behaviours are affected by circadian rhythms or synchrony effects more substantially than others. Future research should continue to seek a balance 
between the ecological validity and generalizability afforded by experience-sampling studies and the controlled internal validity afforded by lab-based experiments (Lin et al., 2020).

Finally, because the measure of opportunity to empathize (seeing another person express an emotion) was also self-reported, we are unable to determine whether chronotype affected people's actual exposure to other people across the course of the day (objective situation selection), people's perception of other people across the course of the day (subjective perception), or both (Rauthmann et al., 20l5).

\section{Conclusion}

While mood and attention have long been known to fluctuate throughout the day, previous research has not examined whether diurnal effects extend to prosocial behaviors in daily life. Within a preregistered, experience-sampling study with a near-representative sample of Americans, morningtype people were slightly more likely to report both prosocial behaviors and empathizing in the morning compared to in the evening. These within-subject effects were largely due to people with morning-chronotypes reporting more social opportunities earlier in the day. Generally, evening-type people were similarly likely to report prosocial behaviors and feeling of empathy in the mornings and evenings. We note, however, that the effect sizes of both the circadian effects and synchrony effects were quite small. This high-powered, within-subject, more representative, and ecologically valid study offers a perspective on diurnal effects on prosocial behaviour that closely reflects what actually occurs in daily life. 


\section{References}

Adan, A., \& Almirall, H. (199I). Horne \& Östberg morningness-eveningness questionnaire: A reduced scale. Personality and Individual Differences, I2(3), 24I-253. https://doi.org/I0.1016/019I-8869(9I)90I I0-W

Arechar, A. A., Kraft-Todd, G. T., \& Rand, D. G. (2017). Turking overtime : how participant characteristics and behavior vary over time and day on Amazon Mechanical Turk. Journal of the Economic Science Association, 3, I-II. https://doi.org//0.1007/s4088I-017-0035-0

Bodenhausen, G. V. (1990). Stereotypes as judgmental heuristics: Evidence of circadian variations in discrimination. Psychological Science, I(5), 319-322. https://doi.org/I0.III I/j. I467-9280.1990.tb00226.x

Brown, S. L., Nesse, R. M., Vinokur, A. D., \& Smith, D. M. (2003). Providing support may be more beneficial than receiving it: Results from a prospective study of mortality. Psychological Science, I4(4), 320-327. https://doi.org/ I0.I I I I/I4679280.14461

Cameron, C. D., Hutcherson, C. A., Ferguson, A. M., Scheffer, J. A., Hadjiandreou, E., \& Inzlicht, M. (2019). Empathy is hard work: People choose to avoid empathy because of its cognitive costs. Journal of Experimental Psychology: General, 148(6), 962-976. https://doi.org//0.1037/xge0000595

Caminada, H., \& Bruijn, F. D. E. (1992). Diurnal variation, morningness-eveningness, and momentary affect. European Journal of Personality, 6(July 1991), 43-69.

Carlson, M., Charlin, V., \& Miller, N. (1988). Positive mood and helping behavior: A test of six hypotheses. Journal of Personality and Social Psychology, 55(2), 21 I-229. https://doi.org/I0.1037/0022-35I4.55.2.2I I

Chebat, J.-C., \& Dubé, L. (1997). Individual differences in circadian variations of consumers' emotional state. Perceptual and Motor Skills, 84, 1075-1086.

Correa, A., Ruiz-Herrera, N., Ruz, M., Tonetti, L., Martoni, M., Fabbri, M., \& Natale, V. (20I7). Economic decision-making in morning/evening-type people as a function of time of day. Chronobiology International, 34(2), 139-147. https://doi.org// 0.1080/07420528.2016.1246455

Dickinson, D. L., \& McElroy, T. (2017). Sleep restriction and circadian effects on social decisions. European Economic Review, 97, 57-7I. https://doi.org/10.1016/j.euroecorev.2017.05.002

Duckworth, A. L., Gendler, T. S., \& Gross, J. J. (2016). Situational strategies for self-control. Perspectives on Psychological Science, II(I), 35-55. https://doi.org//0.1 I77//74569/6/5623247

Emmons, R. A., \& Diener, E. (1986). Situation selection as a moderator of response consistency and stability. Journal of Personality and Social Psychology, 5I(5), I0I3-1019. https://doi.org/I0.1037/0022-35I4.5I.5.1013

Faul, F., Erdfelder, E., Buchner, A., \& Lang, A.-G. (2009). Statistical power analyses using G*Power 3.I: Tests for correlation and regression analyses. Behavior Research Methods, 4 I (4), I I49-II60. https://doi.org/I0.3758/BRM.4I.4.I I 49

Fisher, A. J., Medaglia, J. D., \& Jeronimus, B. F. (2018). Lack of group-to-individual generalizability is a threat to human subjects research. Proceedings of the National Academy of Sciences of the United States of America, II 5(27), E6 I06-E6II 5. https://doi.org/10.1073/pnas. I7II978II5

Gerlach, P., Teodorescu, K., \& Hertwig, R. (2019). The truth about lies: A meta-analysis on dishonest behavior. Psychological Bulletin, I45(I), I-44.

Goldstein, D., Hahn, C. S., Hasher, L., Wiprzycka, U. J., \& Zelazo, P. D. (2007). Time of day, intellectual performance, and behavioral problems in Morning versus Evening type adolescents: Is there a synchrony effect? Personality and Individual Differences, 42(3), 43I-440. https://doi.org/I0.1016/j.paid.2006.07.008

Gunia, B. C., Barnes, C. M. C. M., \& Sah, S. (2014). The morality of larks and owls: Unethical behavior depends on chronotype as well as time of day. Psychological Science, 25(I2), 2272-2274. https://doi.org/I0.1 I77/09567976I454I989

Habashi, M. M., Graziano, W. G., \& Hoover, A. E. (2016). Searching for the prosocial personality: A Big Five approach to linking personality and prosocial behavior. Personality and Social Psychology Bulletin, 42(9), I I77-I 192. 
https://doi.org//0.1177/0146167216652859

Hasler, B. P., Mehl, M. R., Bootzin, R. R., \& Vazire, S. (2008). Preliminary evidence of diurnal rhythms in everyday behaviors associated with positive affect. Journal of Research in Personality, 42, I54I-I550. https://doi.org/10.1016/j.jp.2008.07.012

Heyman, T., Vankrunkelsven, H., Voorspoels, W., White, A., Storms, G., \& Verheyen, S. (2020). When cheating is an honest mistake: A critical evaluation of the matrix task as a measure of dishonesty. Collabra: Psychology, 6(I), 12. https://doi.org// 0.1525/collabra.294

Hossain, M. T., \& Saini, R. (20I4). Suckers in the morning, skeptics in the evening: Time-of-Day effects on consumers' vigilance against manipulation. Marketing Letters, 25(2), 109-12I. https://doi.org//0.1007/s I 1002-013-9247-0

Hwang, J. Y., Kang, S., Gwak, A. R., Park, J., \& Jin, Y. (2016). The associations of morningness - eveningness with anger and impulsivity in the general population. Chronobiology International, 33(2), 200-209. https://doi.org/I0.3109/07420528.2015.II 28947

Job, V., Dweck, C. S., \& Walton, G. M. (2010). Ego depletion-Is it all in your head? Implicit theories about willpower affect self-regulation. Psychological Science, 2 I(II), I686-1693. https://doi.org/I0.I I77/09567976I0384745

Kerkhof, G. A. (1998). The 24-hour variation of mood differs between morning- and evening-type individuals. Perceptual and Motor Skills, 86, 264-266.

Kouchaki, M., \& Smith, I. H. (2014). The morning morality effect: The influence of time of day on unethical behavior. Psychological Science, 25, 95-102. https://doi.org//0.II77/09567976/3498099

Lara, T., Madrid, J. A., \& Correa, Á. (2014). The vigilance decrement in executive function is attenuated when individual chronotypes perform at their optimal time of day. PLoS ONE, 9(2). https://doi.org//0.137//journal.pone.0088820

Lefevor, G. T., Fowers, B. J., Ahn, S., Lang, S. F., \& Cohen, L. M. (2017). To what degree do situational influences explain spontaneous helping behaviour? A meta-analysis. European Review of Social Psychology, 28(I), 227-256. https://doi.org// 0.1080/10463283.2017.1367529

Lin, H., Werner, K., \& Inzlicht, M. (2020). Promises and perils of experimentation: The mutual internal validity problem. https://doi.org/l0.3/234/osf.io/hwubj

Lockwood, P. L., Hamonet, M., Zhang, S. H., Ratnavel, A., Salmony, F. U., Husain, M., \& Apps, M. A. J. (2017). Prosocial apathy for helping others when effort is required. Nature Human Behaviour, I(7), I-10. https://doi.org/I0.1038/s4I562$0|7-0| 3 \mid$

Matchock, R. L., \& Mordkoff, J. T. (2009). Chronotype and time-of-day influences on the alerting, orienting, and executive components of attention. Experimental Brain Research, 192(2), 189-198. https://doi.org/10.1007/s0022I-008-1567-6

Nezlek, J. B., Feist, G. J., Wilson, F. C., \& Plesko, R. M. (200I). Day-to-day variability in empathy as a function of daily events and mood. Journal of Research in Personality, 35(4), 40I-423. https://doi.org/I0.1006/jrpe.200I.2332

Nowack, K., \& Van Der Meer, E. (2018). The synchrony effect revisited : chronotype, time of day and cognitive performance in a semantic analogy task. Chronobiology International, 35(I2), I647-I662. https://doi.org//0.1080/07420528.2018.1500477

Park, C. II, An, S. K., Kim, H. W., Koh, M. J., Namkoong, K., Kang, J. I., \& Kim, S. J. (20I5). Relationships between chronotypes and affective temperaments in healthy young adults. Journal of Affective Disorders, 175, 256-259. https://doi.org/10.1016/j.jad.2015.01.004

Preacher, K. J., Curran, P. J., \& Bauer, D. J. (2004). Simple Intercepts, Simple Slopes, and Regions of Significance in MLR 3Way Interactions. Unpublished, I-5.

Rauthmann, J. F., Sherman, R. A., Nave, C. S., \& Funder, D. C. (20I5). Personality-driven situation experience, contact, and construal: How people's personality traits predict characteristics of their situations in daily life. Journal of Research in Personality, 55, 98-III. https://doi.org/I0.1016/j.jrp.2015.02.003

Riley, E., Esterman, M., Fortenbaugh, F. C., \& DeGutis, J. (2017). Time-of-day variation in sustained attentional control. 
Chronobiology International, 34(7), 993-I00I. https://doi.org/I0.1080/07420528.2017.I30895 I

Roeser, K., McGregor, V. E., Stegmaier, S., Mathew, J., Kübler, A., \& Meule, A. (2016). The Dark Triad of personality and unethical behavior at different times of day. Personality and Individual Differences, 88, 73-77. https://doi.org//0.1016/j.paid.2015.09.002

Schmidt, C., Collette, F., Cajochen, C., \& Peigneux, P. (2007). A time to think: Circadian rhythms in human cognition. Cognitive Neuropsychology, 24(7), 755-789. https://doi.org/I0.1080/02643290701754I58

Schofield, T. P. (2016). Time-of-day effects in implicit racial in-group preferences are likely selection effects, not circadian rhythms. PeerJ, 4, el 947. https://doi.org/10.77/7/peerj.1947

Scholz, U. (2019). It's time to think about time in health psychology. Applied Psychology: Health and Well-Being, I I (2), I73186. https://doi.org/10.1 I I I/aphw.12156

Scollon, C. N., Kim-Prieto, C., \& Diener, E. (2003). Experience sampling: Promises and pitfalls, strengths and weaknesses. Journal of Happiness Studies, 4, 5-34. https://doi.org/https://doi.org/I0.1023/A:1023605205II 5

Smith, I. H., \& Kouchaki, M. (20I4). Does the morning morality effect hold true only for morning people? Psychological Science, 25(I2), 2275-2276. https://doi.org//0.I I77/09567976/4552499

Solomon, N. L., \& Zeitzer, J. M. (2019). The impact of chronotype on prosocial behavior. PLoS ONE, I4(4), e02I6309. https://doi.org/I0.137I/ journal.pone.0216309

Sun, J., Rhemtulla, M., \& Vazire, S. (in press). Eavesdropping on missing data: What are people doing when they miss experience sampling reports? Personality and Social Psychology Bu Lletin. https://doi.org// 0.3 I 234/osf.io/5tcwd

Vranka, M., Frollová, N., Pour, M., Novakova, J., \& Houdek, P. (2019). Cheating customers in grocery stores: A field study on dishonesty. Journal of Behavioral and Experimental Economics, 83, 101484. https://doi.org//0.1016/j.socec.2019.101484

Zhang, C., Smolders, K. C. H. J., Lakens, D., \& IJsselsteijn, W. A. (2018). Two experience sampling studies examining the variation of self-control capacity and its relationship with core affect in daily life. Journal of Research in Personality, 74, I02-II3. https://doi.org/10.1016/j.jrp.2018.03.00I

Zhou, H., \& Fishbach, A. (2016). The pitfall of experimenting on the web: How unattended selective attrition leads to surprising (yet false) research conclusions. Journal of Personality and Social Psychology, I I I (4), 493-504.

https://doi.org//0.1037/pspa0000056 